\title{
Noncommutative Hilbert rings
}

\author{
Algirdas Kaučikas, Vilnius* \\ and \\ Robert Wisbauer, Düsseldorf
}

\begin{abstract}
Commutative rings in which every prime ideal is the intersection of maximal ideals are called Hilbert (or Jacobson) rings. This notion was extended to noncommutative rings in two different ways by the requirement that prime ideals are the intersection of maximal or of maximal left ideals, respectively. Here we propose to define noncommutative Hilbert rings by the property that strongly prime ideals are the intersection of maximal ideals. Unlike for the other definitions, these rings can be characterized by a contraction property: $R$ is a Hilbert ring if and only if for all $n \in \mathbb{N}$ every maximal ideal $\mathfrak{M} \subset R\left[X_{1}, \ldots, X_{n}\right]$ contracts to a maximal ideal of $R$. This definition is also equivalent to $R\left[X_{1}, \ldots, X_{n}\right] / \mathfrak{M}$ being a finitely generated as an $R / \mathfrak{M} \cap R$-module, i.e., a liberal extension. This gives a natural form of a noncommutative Hilbert's Nullstellensatz. The class of Hilbert rings is closed under finite polynomial extensions and under integral extensions.

2000 Mathematics Subject Classification 16S38, 16N60, 16S36, 16S20
\end{abstract}

\section{Introduction}

A commutative ring $R$ is called a Hilbert ring, also Jacobson or JacobsonHilbert ring, if every prime ideal of $R$ is the intersection of maximal ideals. This is obviously equivalent to require that in each factor ring of $R$ the nilradical coincides with the Jacobson radical. Evidently, the class of commutative Hilbert rings is closed under forming factor rings. The interest in this class of rings is based on the following characterizations.

\footnotetext{
*The research of the first author was supported by DAAD (Germany).
} 
For a commutative ring $R$ the following are equivalent:

(a) $R$ is a Hilbert ring;

(b) for each maximal ideal $\mathfrak{M} \subset R[X]$, the intersection $\mathfrak{M} \cap R$ is a maximal ideal in $R$;

(c) every maximal ideal of $R[X]$ contains a monic polynomial;

(d) the polynomial ring $R[X]$ is a Hilbert ring.

The class of commutative Hilbert rings is closed under forming finite polynomial rings. For an integral extension $R \subseteq S$, the ring $S$ is Hilbert if and only if $R$ is a Hilbert ring. The main interest in Hilbert rings in commutative algebra and algebraic geometry is their relation with Hilbert's Nullstellensatz.

The notion was extended to noncommutative rings by replacing "maximal ideal" by maximal left or two-sided ideals. Recall that the Jacobson radical of a ring with unit is defined as intersection of all maximal left (or right) ideals, which is equal to the intersection of all primitive (two-sided) ideals. The Brown-McCoy radical of $R$ is the intersection of all maximal ideals of $R$.

A (noncommutative) ring $R$ is named Jacobson ring by Watters in [12], if every prime ideal is the intersection of maximal left (or right) ideals. This is obviously equivalent to require that prime ideals are the intersection of primitive ideals. As shown in Watters [12], $R$ is a Jacobson ring if and only if the polynomial ring $R[X]$ is a Jacobson ring.

In Watters [13] a ring $R$ is called Brown-McCoy ring if every prime ideal is the intersection of maximal (two-sided) ideals. It follows from [13] that $R$ is a Brown-McCoy ring if and only if the polynomial ring $R[X]$ is a BrownMcCoy ring. Moreover Watters shows that for a Brown-McCoy ring $R$ the contraction of a maximal ideal in $R[X]$ yields a maximal ideal in $R$ and gives an example showing that this contraction property does not imply that $R$ is a Brown-McCoy ring.

Note that the above observations on Jacobson and Brown-McCoy rings are subsumed in Theorem 5 of Ferrero-Parmenter [4].

A ring $R$ is a Jacobson (Brown-McCoy) ring if and only if in each factor ring of $R$ the prime radical coincides with the Jacobson (Brown-McCoy) radical.

In any PI ring $R$ primitive ideals are maximal and hence such a ring is a Jacobson ring if and only if it is a Brown-McCoy ring. In this case also the Jacobson and Brown-McCoy radical of $R[X]$ coincide. These rings are studied by Amitsur and Procesi in [1] and [10].

In this article we extend the notion of commutative Hilbert rings to any noncommutative rings by restricting the requirements to a special class of 
prime ideals, the strongly prime ideals. These are related to strongly prime rings which can be characterized by the fact that their central closures (in the sense of Martindale) are simple rings. We give a characterization of Hilbert rings in terms of a contraction property in the rings of polynomials, and the class of Hilbert rings is the largest class of rings having this property. Furthermore, we characterize Hilbert rings in terms of monic polynomials, show that the class of Hilbert rings is closed under finite polynomial and integral extensions, and obtain a natural symmetric form of a general Hilbert's Nullstellensatz for Hilbert rings.

All rings in this paper are associative with identity element which should be preserved by ring homomorphisms. By an ideal of the ring we shall understand a two-sided ideal. $A \subset B$ means that $A$ is proper subset of $B$. Throughout $R\left[X_{1}, \ldots, X_{n}\right]$ will denote the polynomial ring with $n$ commuting indeterminates, which also commute with the elements from the ring $R$.

\section{Strongly prime ideals}

Let $R$ be a prime ring, $Q(R)$ its central closure, and $F(R)$ the extended centroid of the ring $R$, which is the centre of $Q(R)$. As shown in [14, Section 32], $Q(R)$ may be understood as self-injective hull of $R$ as $R \otimes_{\mathbb{Z}} R^{o}$-module. Any element $\varphi \in F(R)$ may be represented as an $R$-bimodule homomorphism $\varphi: I \rightarrow R$ for some nonzero ideal $I \subseteq R$.

A prime ring $R$ is called strongly prime if its central closure $Q(R)$ is a simple ring. Various characterizations of strongly prime rings are given, e.g., in $[14,35.6]$ and $[8$, Theorem 2.1].

Let $\phi: R \rightarrow S$ be a ring homomorphism. Then $S$ is an $R$-bimodule and we write $r s$ and $s r$ instead of $\phi(r) s$ and $s \phi(r)$ for $r \in R, s \in S$. Let $Z_{S}(R)=\{x \in S \mid r x=x r, \forall r \in R\}$ be the set of $R$-centralizing elements of the ring $S$.

We call $\phi$ a centred homomorphism and $S$ a centred extension of $R$ via $\phi$, provided $S=R Z_{S}(R)$. This means that $s=\sum_{k} r_{k} x_{k}$ for each element $s \in S$ with some $r_{k} \in R$ and $x_{k} \in Z_{S}(R)$. If $Z_{S}(R)$ is commutative, then centred extensions are called central extensions. Rings and their centred homomorphisms form a category, known as Procesi category. A centred extension $R \subseteq S$ is called a liberal extension if $S$ is finitely generated as a canonical $R$ module.

An ideal $\mathfrak{p} \subset R$ is called strongly prime if the factor $\operatorname{ring} R / \mathfrak{p}$ is a strongly prime ring. Clearly, maximal ideals are strongly prime. It is well known that 
in PI rings any prime ideal is strongly prime. Since not each prime ring has a simple central closure, prime ideals are not necessarily strongly prime. The following lemma recalls some crucial properties.

Lemma 2.1. Let $\phi: R \rightarrow S$ be a centred homomorphism.

(1) Assume $S$ to be a simple ring. Then:

(i) The kernel of $\phi$ is a strongly prime ideal in $R$.

(ii) If $\phi$ is injective, there is a unique extending ring homomorphism $Q(R) \rightarrow S$ which maps the center of $Q(R)$ into the center of $S$.

(2) Assume $R \subseteq S$ to be a liberal extension. Then for any prime ideal $\mathfrak{p}$ in $R$ there is a prime ideal ideal $\mathfrak{P}$ in $S$ with $\mathfrak{P} \cap R=\mathfrak{p}$ (lying over).

Proof. (1)(i) follows from the characterizations of strongly prime rings (see [8, Theorem 2.1]). (ii) follows from Amitsur [2, Theorem 18].

(2) This is shown in [11, Theorem 4.1].

The intersection of all strongly prime ideals of the ring $R$ is called the strongly prime radical and we denote it by $\operatorname{Sp}(R)$. Let $\operatorname{BMc}(R)$ denote the Brown-McCoy radical of the given ring $R$. These two radicals are closely related.

Theorem 2.2. In any nonzero ring $R$,

$$
\operatorname{Sp}(R)=\bigcap_{n \geq 1}\left(R \cap \operatorname{BMc}\left(R\left[X_{1}, \ldots, X_{n}\right]\right)\right)
$$

Proof. If $a \in R$ does not belong to some maximal ideal $\mathfrak{M} \subset R\left[X_{1}, \ldots . X_{n}\right]$, then $a \notin \mathfrak{M} \cap R=\mathfrak{p}$. Clearly $R \rightarrow R\left[X_{1}, \ldots, X_{n}\right] / \mathfrak{M}$ is a centred extension and, by $2.1, \mathfrak{p} \subset R$ is a strongly prime ideal. So $a \notin \operatorname{Sp}(R)$.

If $a \notin \mathfrak{p}$ for some strongly prime ideal $\mathfrak{p} \subset R$, then in the central closure $Q(R / \mathfrak{p})$, a simple ring, we have an expression

$$
a_{1} \varphi_{1}+\cdots+a_{n} \varphi_{n}=1
$$

where $a_{1}, \ldots, a_{n} \in(a)=R a R$, and $\varphi_{1}, \ldots, \varphi_{n}$ are from the extended centroid of the ring $R / \mathfrak{p}$, which is the centre of $Q(R / \mathfrak{p})$. So we obtain a centred homomorphism

$$
\phi: R\left[X_{1}, \ldots, X_{n}\right] \rightarrow Q(R / \mathfrak{p}), \quad X_{k} \mapsto \varphi_{k} \text { for } 1 \leq k \leq n
$$


Evidently, the polynomial $a_{1} X_{1}+\cdots+a_{n} X_{n}-1$ is in the kernel of $\phi$. If $\mathfrak{M} \subset R\left[X_{1}, \ldots, X_{n}\right]$ is a maximal ideal containing $\operatorname{ker} \phi$, then $a \notin \mathfrak{M}$, and $a \notin \operatorname{BMc}\left(R\left[X_{1}, \ldots, X_{n}\right]\right)$.

Notice that the proof of the theorem also follows by Corollary 4.7 and Theorem 4.12 in [5].

In terms of elements, $a \in \operatorname{Sp}(R)$ if and only if for any $a_{1}, \ldots, a_{n} \in(a)$, the ideal in $R\left[X_{1}, \ldots, X_{n}\right]$, generated by the polynomial $a_{1} X_{1}+\cdots+a_{n} X_{n}-1$, contains $1_{R}$ (see [8, Theorem 3.2]).

Theorem 2.3. Let $\mathfrak{M} \subset R\left[X_{1}, \ldots, X_{n}\right]$ be a maximal ideal and $\mathfrak{p}=\mathfrak{M} \cap R$. Then the following are equivalent:

(a) $\mathfrak{p}$ is a maximal ideal in $R$;

(b) there exists a monic polynomial $f \in R[T]$ such that $f\left(X_{k}\right) \in \mathfrak{M}$ for all $1 \leq k \leq n$;

(c) $R / \mathfrak{p} \subseteq R\left[X_{1}, \ldots, X_{n}\right] / \mathfrak{M}$ is a liberal extension.

Proof. $(a) \Rightarrow(b)$ Let $\mathfrak{p} \subset R$ be a maximal ideal. So

$$
\mathcal{A}=R / \mathfrak{p} \hookrightarrow R\left[X_{1}, \ldots, X_{n}\right] / \mathfrak{M}=\mathcal{S}
$$

is a central extension of a simple ring. $\mathcal{S}=\mathcal{A}\left[x_{1}, \ldots, x_{n}\right]$, where $x_{1}, \ldots, x_{n}$ are the images of $X_{1}, \ldots, X_{n}$ respectively, and these elements are from the centre of $\mathcal{S}$. If the field $F$ is the centre of $\mathcal{A}$, and $F\left[x_{1}, \ldots, x_{n}\right]$ the subring of $\mathcal{S}$, generated by $F$ and $x_{1}, \ldots x_{n}$, then we have the canonical surjective homomorphism

$$
\phi: \mathcal{A} \otimes_{F} F\left[x_{1}, \ldots, x_{n}\right] \longrightarrow \mathcal{S} .
$$

By the definition of $F\left[x_{1}, \ldots, x_{n}\right]$, the restriction of the homomorphism $\phi$ to this ring is a monomorphism. Because $\mathcal{A}$ is a central simple $F$-algebra, $\phi$ is a monomorphism by standard properties of central simple algebras. So $\phi$ is an isomorphism. Now we see that the $\operatorname{ring} \mathcal{L}=F\left[x_{1}, \ldots, x_{n}\right]$, which is a finitely generated $F$-algebra, is a field - again by the ideal structure of the tensor product of central simple algebras. By one of the versions of Hilbert's Nullstellensatz (e.g., Theorem 4.19 in [3]), $\mathcal{L}$ is a finite algebraic extension of $F$. So the elements $x_{k} \in \mathcal{L}, 1 \leq k \leq n$, are algebraic over the field $F$. Thus there exist monic polynomials $g_{k} \in F[T]$ such that $g_{k}\left(x_{k}\right)=0$. Lifting the product $g_{1} \cdots g_{n}$ to a monic polynomial $f \in R[T]$, we obtain that $f\left(X_{k}\right) \in$ $\mathfrak{M}, 1 \leq k \leq n$. 
Evidently, (c) follows from (b).

$(c) \Rightarrow(a)$ The simple ring $\mathcal{S}$ is a liberal extension of the ring $\mathcal{A}$. By 2.1(2), this implies that $\mathcal{A}$ is also a simple ring, so the ideal $\mathfrak{p}=\mathfrak{M} \cap R$ is maximal.

\section{Noncommutative Hilbert rings}

We call a ring $R$ a Hilbert ring if every strongly prime ideal is the intersection of maximal ideals. This is obviously equivalent to require that in each factor ring of $R$, the strongly prime radical coincides with the Brown-McCoy radical. It is also clear that any factor ring of a Hilbert ring is again Hilbert.

Since in commutative rings strongly prime ideals are precisely prime ideals, this extends the notion of commutative Hilbert rings. Moreover, the class of Hilbert rings contains the Brown-McCoy rings considered in [13] and also the Jacobson PI rings considered in [1] and [10]. By Theorem 2.5 in [8], strongly prime ideals are preserved under Morita equivalences, and so the property to be a Hilbert ring is preserved under Morita equivalences.

The importance of this notion is based on the following characterizations.

Theorem 3.1. For any ring $R$ the following are equivalent:

(a) $R$ is a Hilbert ring;

(b) for each $n \in \mathbb{N}$ and any maximal ideal $\mathfrak{M}$ of $R\left[X_{1}, \ldots, X_{n}\right]$, the contraction $\mathfrak{M} \cap R$ is a maximal ideal of $R$;

(c) for each $n \in \mathbb{N}$ and every maximal ideal $\mathfrak{M}$ of $R\left[X_{1} \ldots, X_{n}\right]$, there exists a monic polynomial $f \in R[T]$, such that $f\left(X_{k}\right) \in \mathfrak{M}$, for $1 \leq k \leq n$;

(d) for each $n \in \mathbb{N}$ and every maximal ideal $\mathfrak{M} \subset R\left[X_{1}, \ldots, X_{n}\right]$, the extension $R / \mathfrak{M} \cap R \hookrightarrow R\left[X_{1}, \ldots, X_{n}\right] / \mathfrak{M}$ is liberal;

(e) the polynomial ring $R[X]$ is a Hilbert ring.

Proof. $(a) \Rightarrow(b)$ Let $\mathfrak{M} \subset R\left[X_{1}, \ldots, X_{n}\right]$ be a maximal ideal. Then, by Remark 4.5 in [5], $\mathfrak{p}=\mathfrak{M} \cap R$ is a strongly prime ideal in $R$ and the intersection of all nonzero prime ideals of $R / \mathfrak{p}$ (i.e., the pseudo-radical of $R / \mathfrak{p}$ ) is nonzero. However, if $R$ is a Hilbert ring, the intersection of all maximal ideals of $R / \mathfrak{p}$ has to be zero. This implies that zero is a maximal ideal in $R / \mathfrak{p}$ and hence $\mathfrak{p}$ is a maximal ideal in $R$.

$(b) \Rightarrow(a)$ Let $\mathfrak{M}_{\alpha}, \alpha \in \mathcal{I}_{n}$, be the family of maximal ideals of the polynomial ring $R\left[X_{1}, \ldots, X_{n}\right]$. We have

$$
R \cap \operatorname{BMc}\left(R\left[X_{1}, \ldots, X_{n}\right]\right)=R \cap\left(\bigcap_{\alpha} \mathfrak{M}_{\alpha}\right)=\bigcap_{\alpha}\left(R \cap \mathfrak{M}_{\alpha}\right)=\operatorname{BMc}(R),
$$


because all ideals $R \cap \mathfrak{M}$ are maximal in $R$, and each maximal ideal of $R$ can be obtained in this way. So, by Theorem $2.2, \operatorname{Sp}(R)=\operatorname{BMc}(R)$.

The equivalence $(b) \Leftrightarrow(c) \Leftrightarrow(d)$ follows easily from Theorem 2.3.

$(d) \Rightarrow(e)$ Take a maximal ideal $\mathfrak{M} \subset R\left[X, X_{1}, \ldots, X_{n}\right]$. By $(d)$, the extension $R / \mathfrak{M} \cap R \hookrightarrow R\left[X, X_{1}, \ldots, X_{n}\right] / \mathfrak{M}$ is liberal, so the extension

$$
R[X] / \mathfrak{M} \cap R[X] \hookrightarrow R\left[X, X_{1}, \ldots, X_{n}\right] / \mathfrak{M}
$$

is also liberal. Hence $R[X]$ satisfies $(d)$ and so it is a Hilbert ring.

Since factor rings of the Hilbert rings are again Hilbert, $(e) \Rightarrow(a)$.

Now we obtain the following corollary which may be considered as a general symmetric form of Hilbert's Nullstellensatz, see [3, Theorem 4.19].

Corollary 3.2. If $R$ is a Hilbert ring, then the ring $R\left[X_{1}, \ldots, X_{n}\right]$ is also a Hilbert ring. In this case, for each maximal ideal $\mathfrak{M} \subset R\left[X_{1}, \ldots, X_{n}\right]$, the factor ring $R\left[X_{1}, \ldots, X_{n}\right] / \mathfrak{M}$ is a central liberal extension of the simple ring $R / \mathfrak{M} \cap R$.

The following definition of integral homomorphisms and integral extensions in a Procesi category and their main properties are considered in [7]. A centred ring homomorphism $\phi: R \rightarrow S$ is called an integral homomorphism if every finite subset $\left\{s_{1}, \ldots, s_{n}\right\} \subseteq S$ is contained in some subring $A \subseteq S$ which is a liberal extension of the ring $\phi(R)$, i.e., as an $R$-module $A$ is generated by a finite set of $R$-centralizing elements. In this case $S$ is called an integral extension of $R$ via $\phi$. In the commutative case this definition is equivalent to the classical definition of an integral homomorphism. Evidently, liberal extensions of rings are integral, and each integral extension is the inductive limit of liberal extensions. It is clear that an integral extension of a field is precisely a locally finite algebras over this field. The following fundamental properties are shown in the theorems 9, 10 and 11 of [7].

Lemma 3.3. Let $R \subset S$ be a centred integral extension.

(1) For any strongly prime ideal $\mathfrak{p}$ in $R$, there exists a strongly prime $\mathfrak{q}$ in $S$ such that $\mathfrak{q} \cap R=\mathfrak{p}$ (lying over).

(2) If $R$ is simple and $S$ is strongly prime, then $S$ is simple.

(3) Consider ideals $\mathfrak{q} \subseteq A$ in $S$ such that $\mathfrak{q} \cap R=A \cap R$. If $\mathfrak{q}$ is a strongly prime ideal then $\mathfrak{q}=A$ (incomparability).

This allows us to prove our final result generalising the commutative case. 
Theorem 3.4. Let $R \subset S$ be an integral extension of rings. Then $S$ is a Hilbert ring if and only if $R$ is a Hilbert ring.

Proof. Let $S$ be a Hilbert ring and $\mathfrak{p} \subset R$ a strongly prime ideal. By 3.3(1), there exists a strongly prime ideal $\mathfrak{q} \subset S$ lying over $\mathfrak{p}$. So $\mathfrak{q}$ is an intersection of maximal ideals in $S$. By (1) and (3) in 3.3, the contraction of a maximal ideal in $S$ is a maximal ideal in $R$. Hence $\mathfrak{p}$ is the intersection of maximal ideals and $R$ is a Hilbert ring.

Let $R$ be a Hilbert ring. If $\mathfrak{q} \subset S$ is a strongly prime ideal, then $\mathfrak{q} \cap R$ is a strongly prime ideal in $R$. Going to factor rings, we reduce the proof to the case of an integral extensions of strongly prime rings. Take a nonzero element $s \in S$. By 3.3(3), the ideal $(s)$ intersects nontrivially with $R$. So we can find a maximal ideal $\mathfrak{m} \subset R$ which does not contain $(s) \cap R$. Now, by 3.3(2), there is a maximal ideal in $S$ lying over $\mathfrak{m}$, which does not contain $(s)$. This means, that the intersection of maximal ideals in $S$ is zero, so $S$ is a Hilbert ring.

\section{References}

[1] Amitsur, S.A., Procesi, C., Jacobson rings and Hilbert algebras with polynomial identities, Ann. Mat Pura Applicata 71 (1966), 61-72.

[2] Amitsur, S.A., On rings of quotients, Symp. Mathematica 8 (1972), 149164.

[3] Eisenbud, D., Commutative Algebra with a View Toward Algebraic Geometry, Springer-Verlag 1995.

[4] Ferrero, M., Parmenter M.M., A note on Jacobson rings and polynomial rings, Proc.Amer.Math.Soc. 105 (1989), 281 - 286.

[5] Ferrero, M., Wisbauer, R., Unitary strongly prime rings and related radicals, J. Pure Appl. Algebra 181 (2003), 209 - 226.

[6] Goldman, O., Hilbert rings and the Hilbert Nullstellensatz, Math. Zeit. 54 (1951), 136-140.

[7] Kaučikas, A., On centred and integral homomorphisms, Lith. Math.J. 37(3) (1997), 264-268.

[8] Kaučikas, A., Wisbauer, R., On strongly prime rings and ideals, Comm. Algebra 28 (2000), 5461-5473. 
[9] Krempa, J., On radical properties of polynomial rings, Bull. Acad. Polon. Sci., Sér. Sci. Math. Astron. Phys. 20 (1972), 545-548.

[10] Procesi, C., Noncommutative Jacobson rings, Ann. Scuola Norm. Sup. Pisa 21 (1967), 281 - 290.

[11] Robson, J.C., Small, L.W., Liberal extensions, Proc. London Math. Soc.(3), 42 (1981), 87-103.

[12] Watters, J.F., Polynomial extensions of Jacobson rings, J. Algebra 36 (1975), $302-308$.

[13] Watters, J.F., The Brown-McCoy radical and Jacobsosn Rings, Bull. Acad. Polon. Sci., Sér. Sci. Math. Astron. Phys. 24 (1976), 91-99.

[14] Wisbauer, R., Modules and Algebras: Bimodule Structure and Group Action on Algebras, Pitman Monographs and Surveys in Pure and Applied Mathematics, vol 81, Addison Wesley, Longman 1996.

\section{Addresses}

Vilnius Pedagogical University, Studentu 39, Vilnius, 2034, Lithuania, e-mail: al.kauchikas@vpu. It

Heinrich-Heine-University, 40225 Düsseldorf, Germany, e-mail: wisbauer@math.uni-duesseldorf.de 